\title{
Accelerators for HEP: Challenges and R\&D
}

\section{Vladimir SHILTSEV (Fermilab)}

ICHEP'2020

3 August 2020 - Prague (virtual)

This manuscript has been authored by Fermi Research Alliance, LLC under Contract No. DE-AC02-07CH11359 with the U.S. Department of Energy, Office of Science, Office of High Energy Physics. 


\section{Accelerator R\&D}

\section{is needed to address feasibility of}

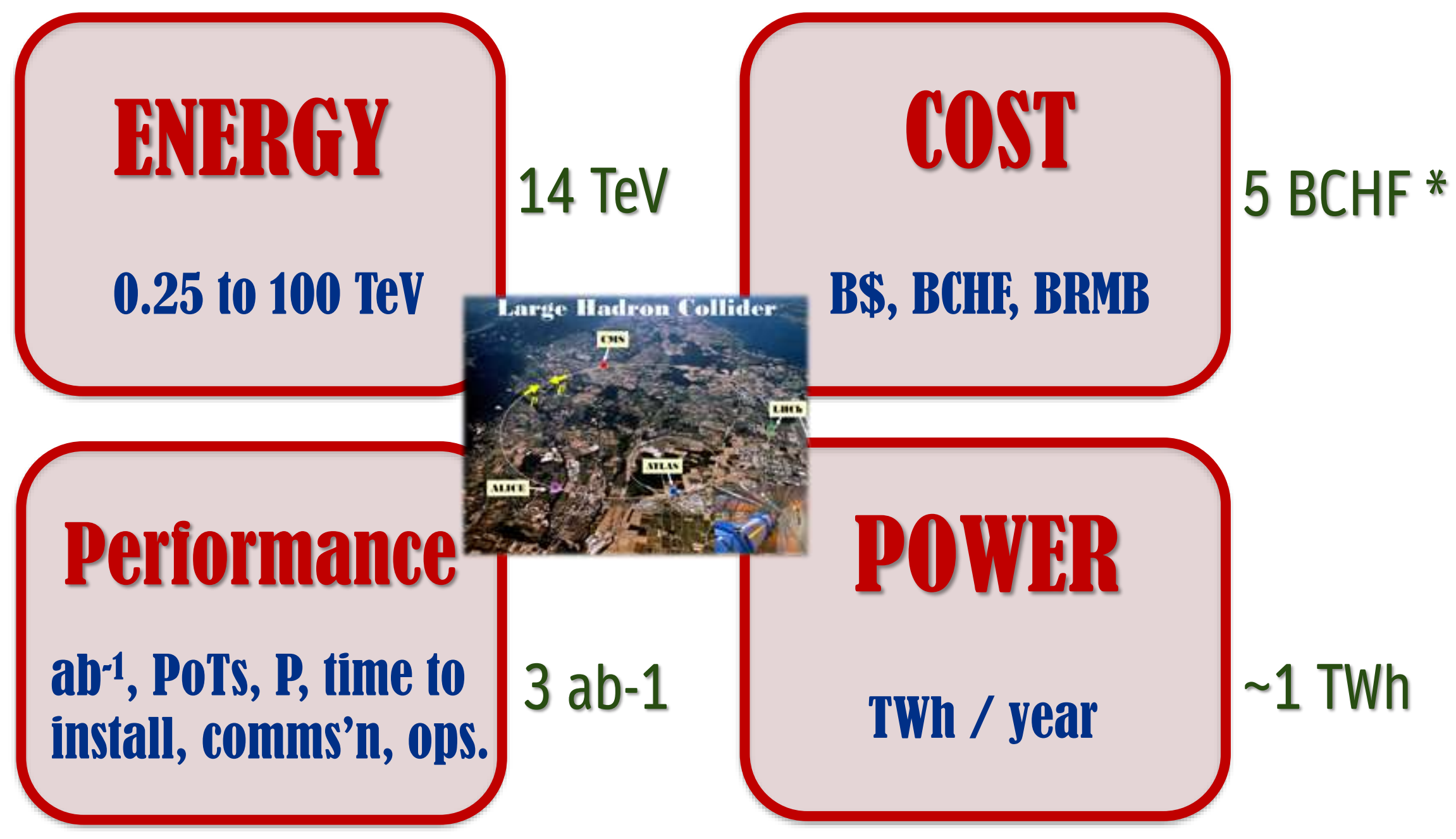


Leading Accelerator Facilities for Neutrino Physics Research

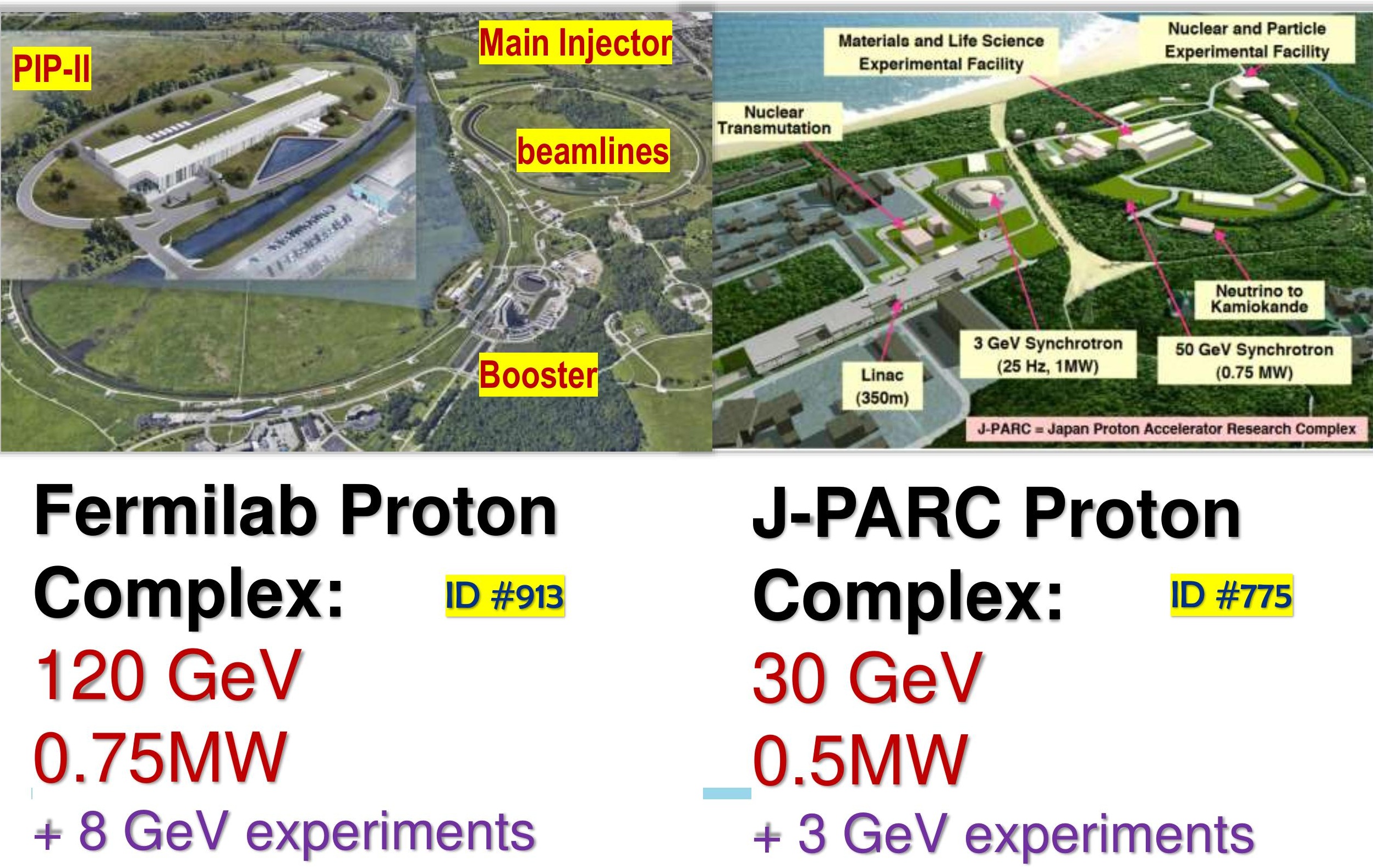




\section{Fermilab and J-PARC Power Upgrades}

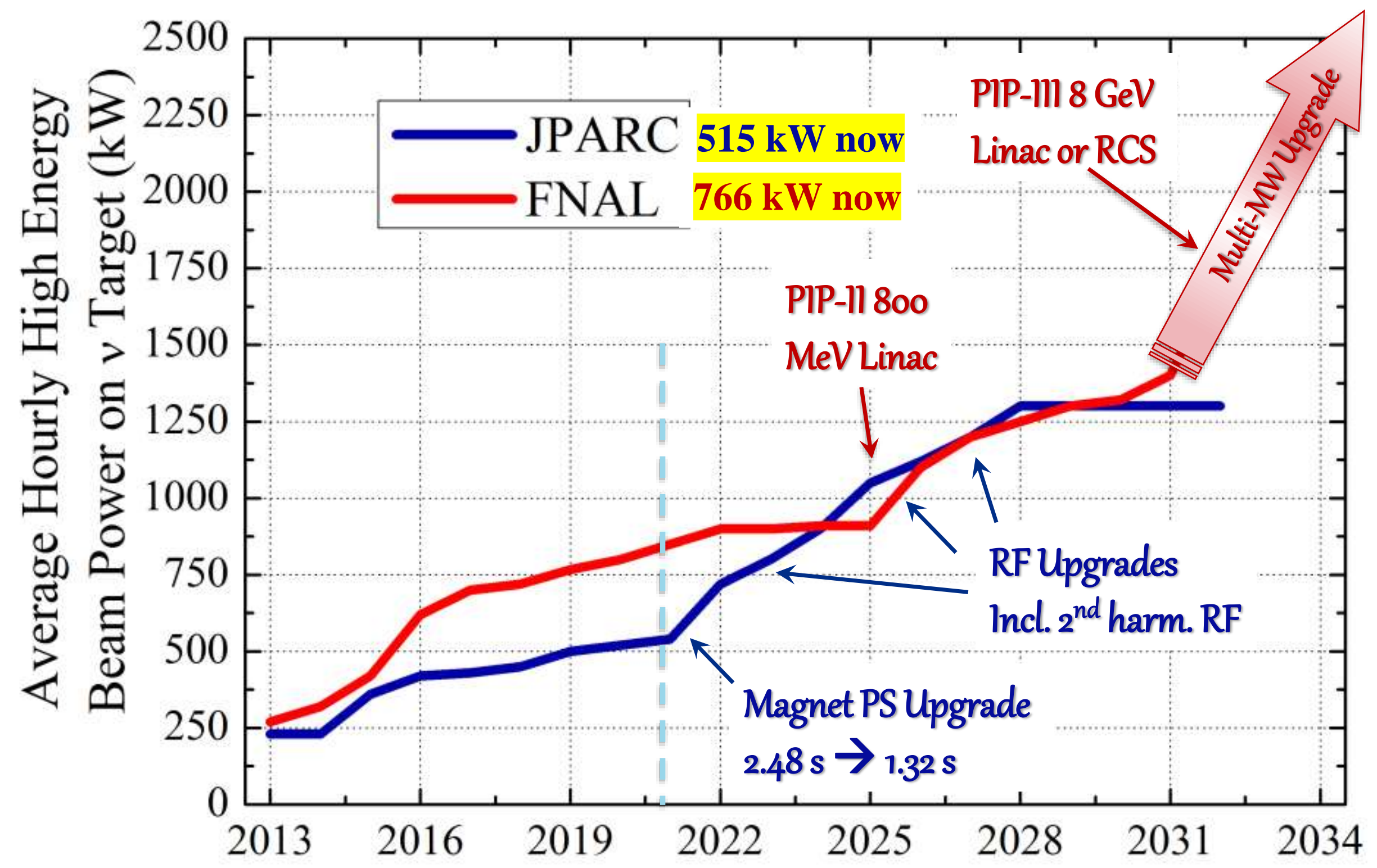




\section{Integrable Optics Test Accelerator (FNAL)}

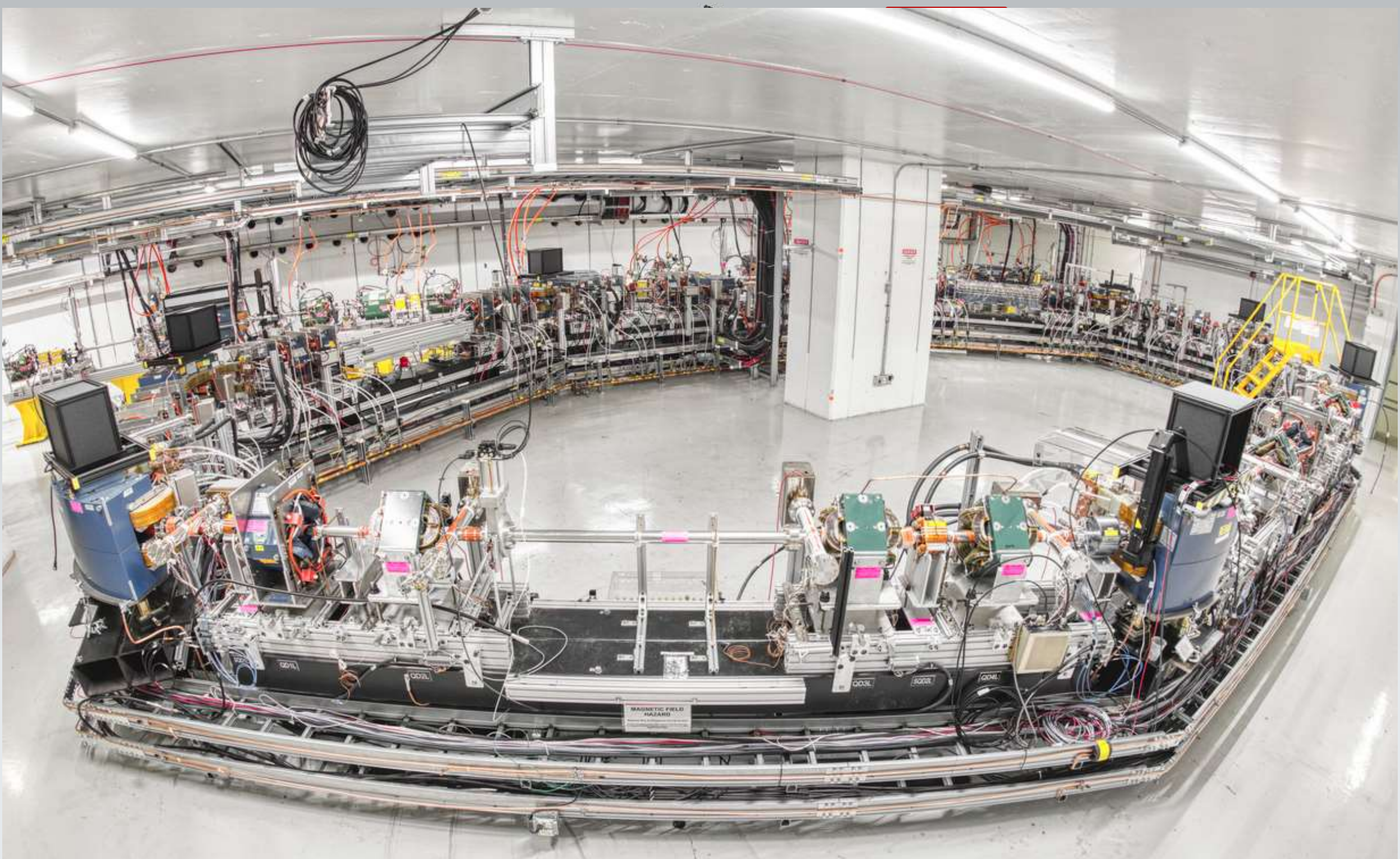


Challenge \#2: Targets, Horns, Beam Windows

- Existing $v$ targets and horns are good to $\sim 0.8$ MW, MW and multi-MW targets are under development

- Issues depend on pulse structure and include radiation damage and thermal shock -waves

- R\&D program to study material properties, new forms (foams, fibers, etc), new target designs (rotating, etc)

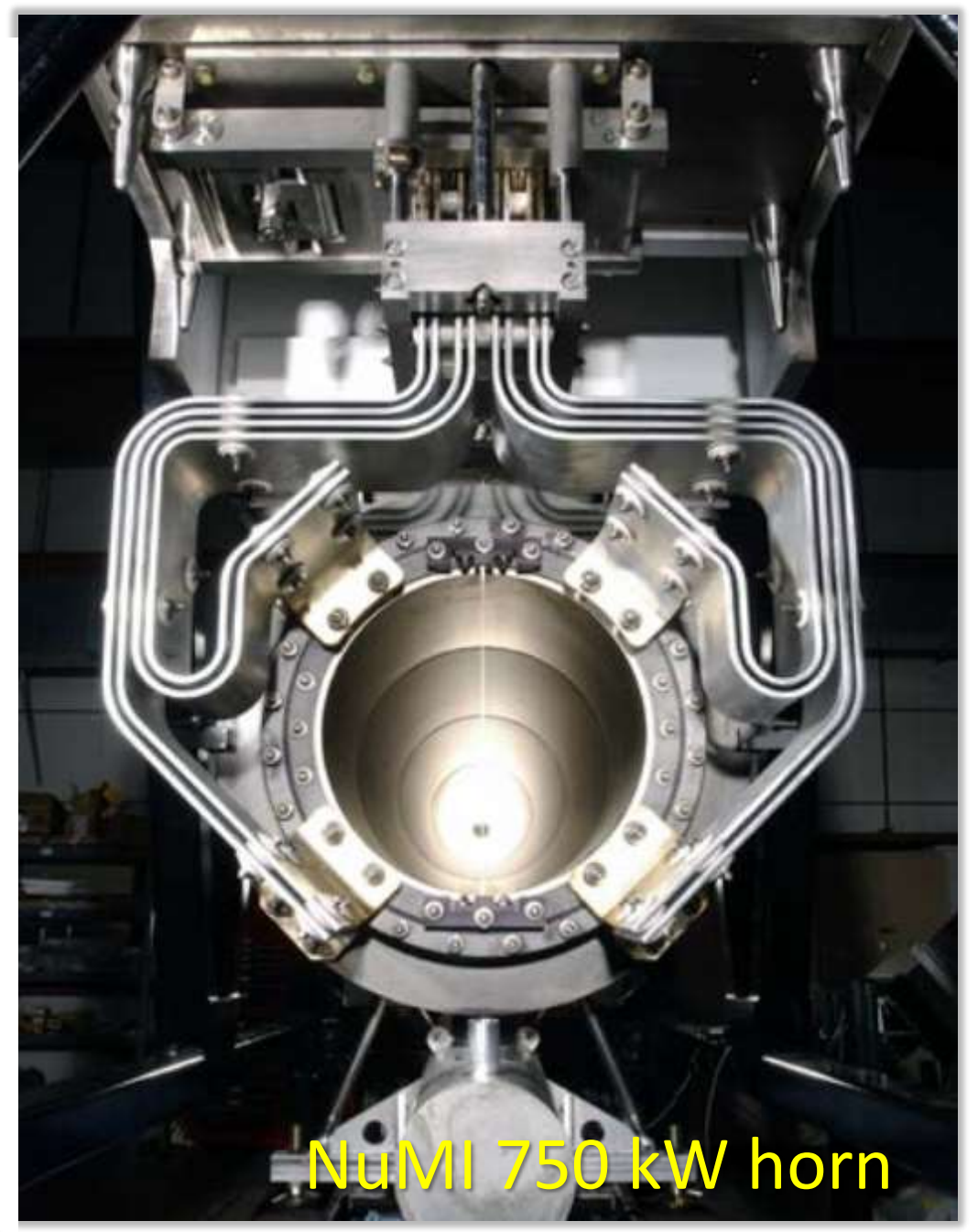




\section{Proposed Facilities for Neutrino Research}

Protvino-to-ORKA:

$70 \mathrm{GeV}, 90$ (450) kW

$L=2590 \mathrm{~km}, E_{v} \sim 5 \mathrm{GeV}$

ENUBET at CERN-SPS:

$400 \mathrm{GeV}, 510 \mathrm{~kW}$

$L=40 \mathrm{~m}, E_{v} \sim 0.5-3.5 \mathrm{GeV}$

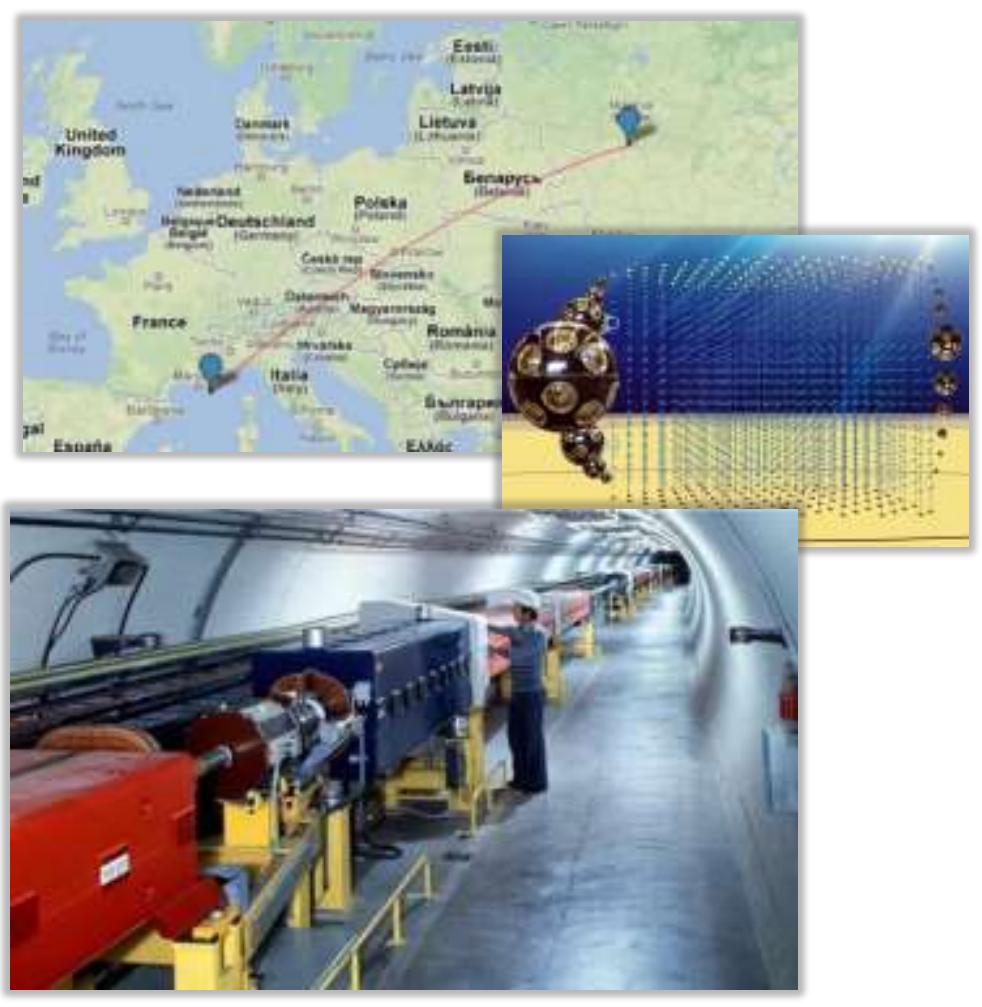

ESS Neutrino Superbeams:

$2 \mathrm{GeV}, 5 \mathrm{MW}$

$L=540 \mathrm{~km}, E_{v} \sim 0.3 \mathrm{GeV}$

Challenges:

$S C$ in the accumulator ring

${ }_{8} 5 \mathrm{MW}$ neutrino target (not spallation)

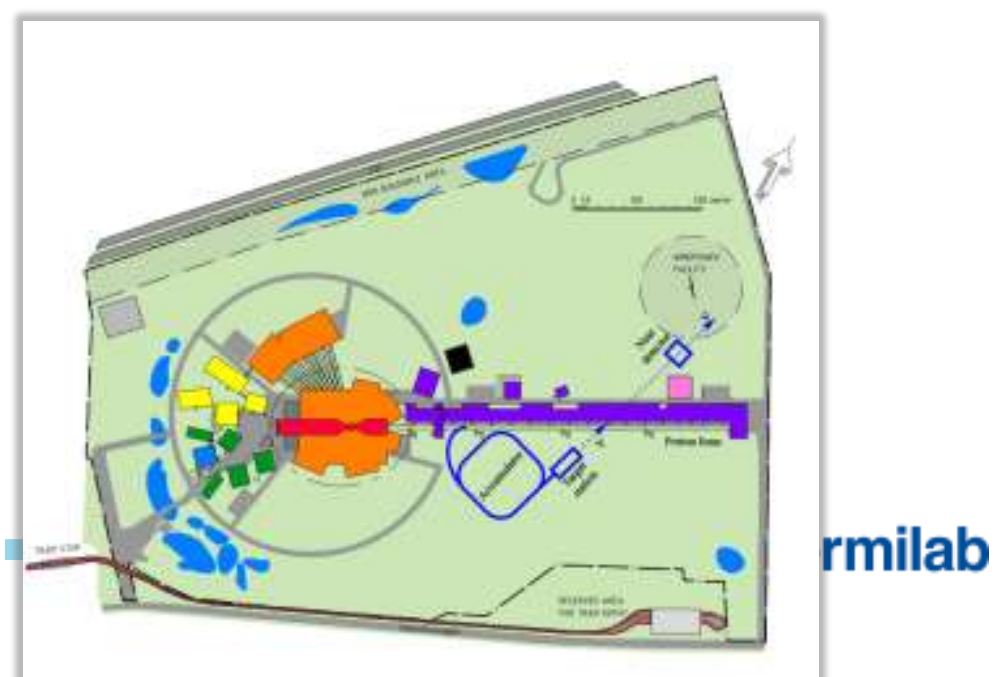




\section{vSTORM}

\section{SPS at CERN :}

$E \equiv 100 \mathrm{GeV} P$ beam $=\mathbf{1 5 6 k W}$

$410^{13} p+$ per pulse

$T_{\text {cycle }}=3.6 \mathrm{~s}, 2 \times 10 \mu \mathrm{s}$ (fast extr.)

$\mu \pm$ beams $1 \mathrm{GeV} / \mathrm{c}-6 \mathrm{GeV} / \mathrm{c}$ momentum spread of $16 \%$

\section{Challenges:}

a) $300 \mu \mathrm{mrad}$ emittance $\rightarrow 0.5$ dia magnets;

b) survival $\sim 60 \%$ after 100 turns for $\delta P / P \sim 10 \%$

\section{Syngergy w. $v$-Factory}

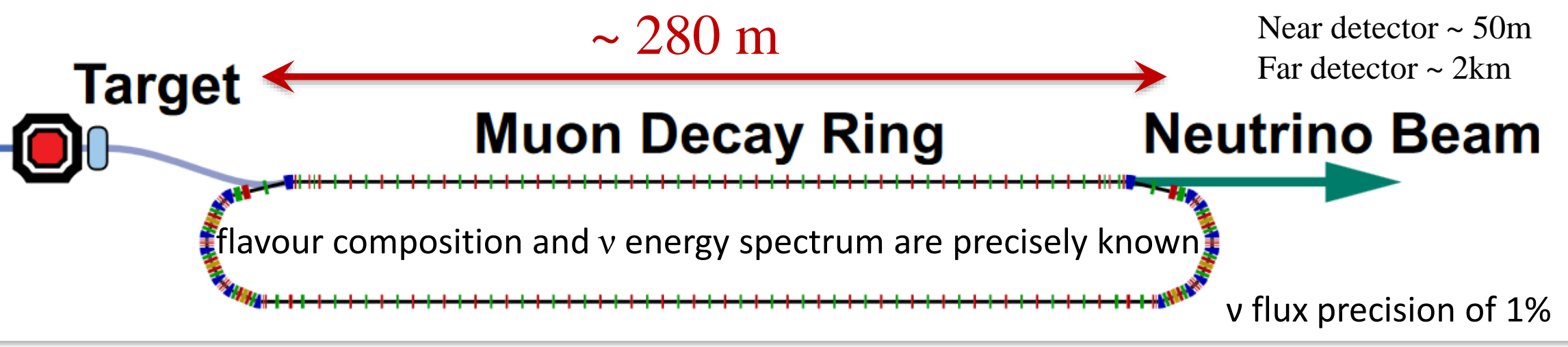




\section{Colliders: (Too) Many on the Table}

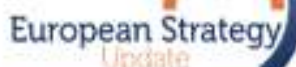

\section{Strategy Update}

\section{High-priority future initiatives}

Map of possible future
facilities submitted as
input to the Strategy
Update

Possible scenarios of future colliders

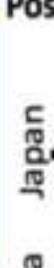

if

f

5.987 vears $1.5 a^{-2}$

$100 \mathrm{~km}$ tunnel

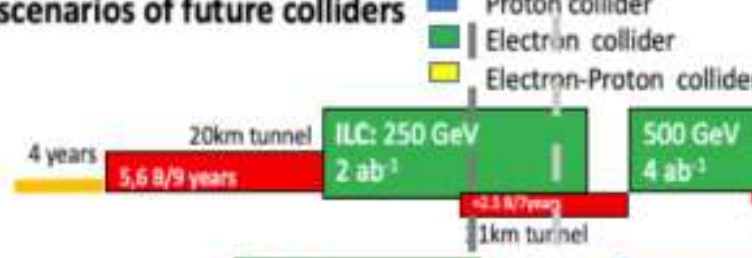

Construction/Transformation: heights of box construction cost/vear = Preparation

1 TeV

$=4.5 .4 \mathrm{ab}^{2}$

$5 p p C:=F C C-b h$

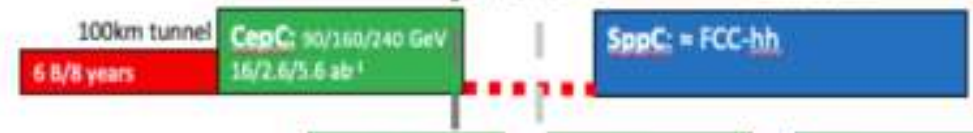

\section{5 years $11 \mathrm{~km}$ tunnel cucis $390 \mathrm{GN}, 1.5 \mathrm{TeV}$}

$|5,6 \mathrm{~B} / 5 \mathrm{y}| \frac{2.5 \mathrm{ab}:}{7,38 / 5 \mathrm{y}}$

29 (m) tunnê

$5 \mathrm{ab}^{2}$

$50 \mathrm{~km}$ tunnel
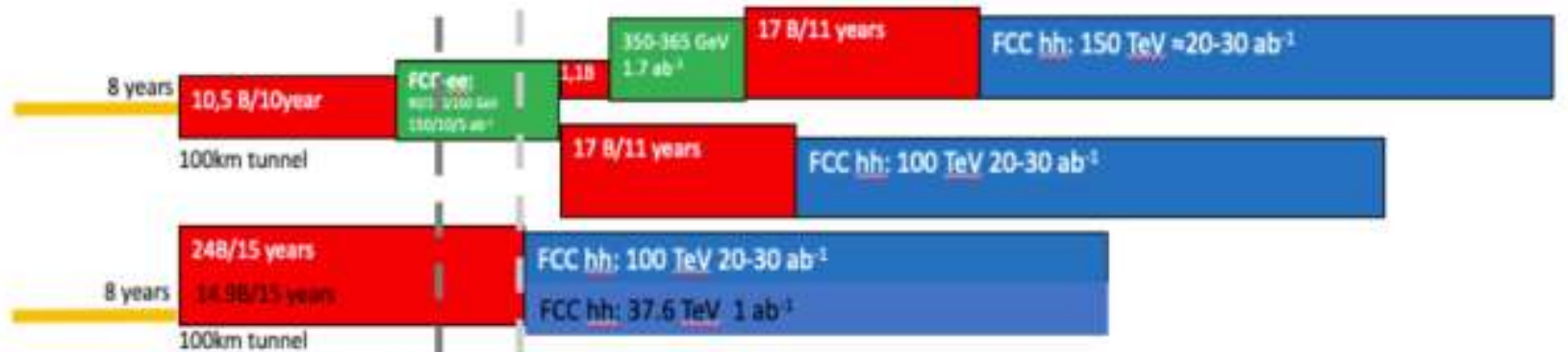
f FCC hh: $37.6 \mathrm{TeV} 1 \mathrm{ab}$

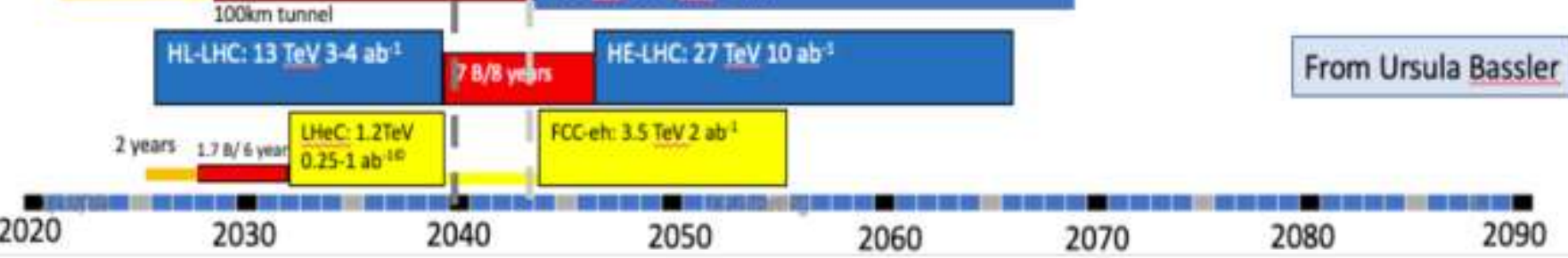




\section{Colliders: 16 Options at Snowmass'21}

Day 1: https://indico.fnal.gov/event/43871/ Day 2: https://indico.fnal.gov/event/43872/

Machine parameters, technical maturity \& timeframe

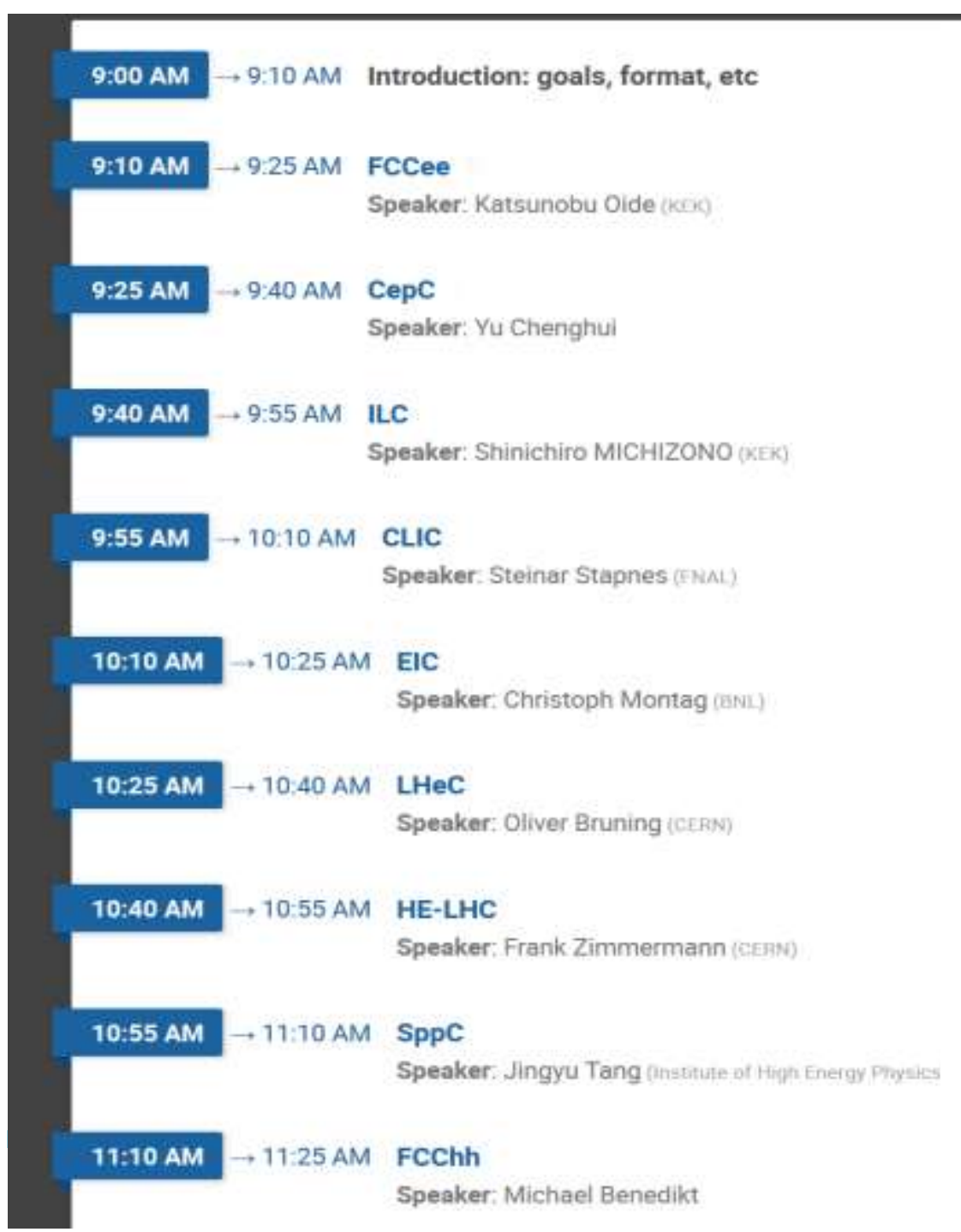

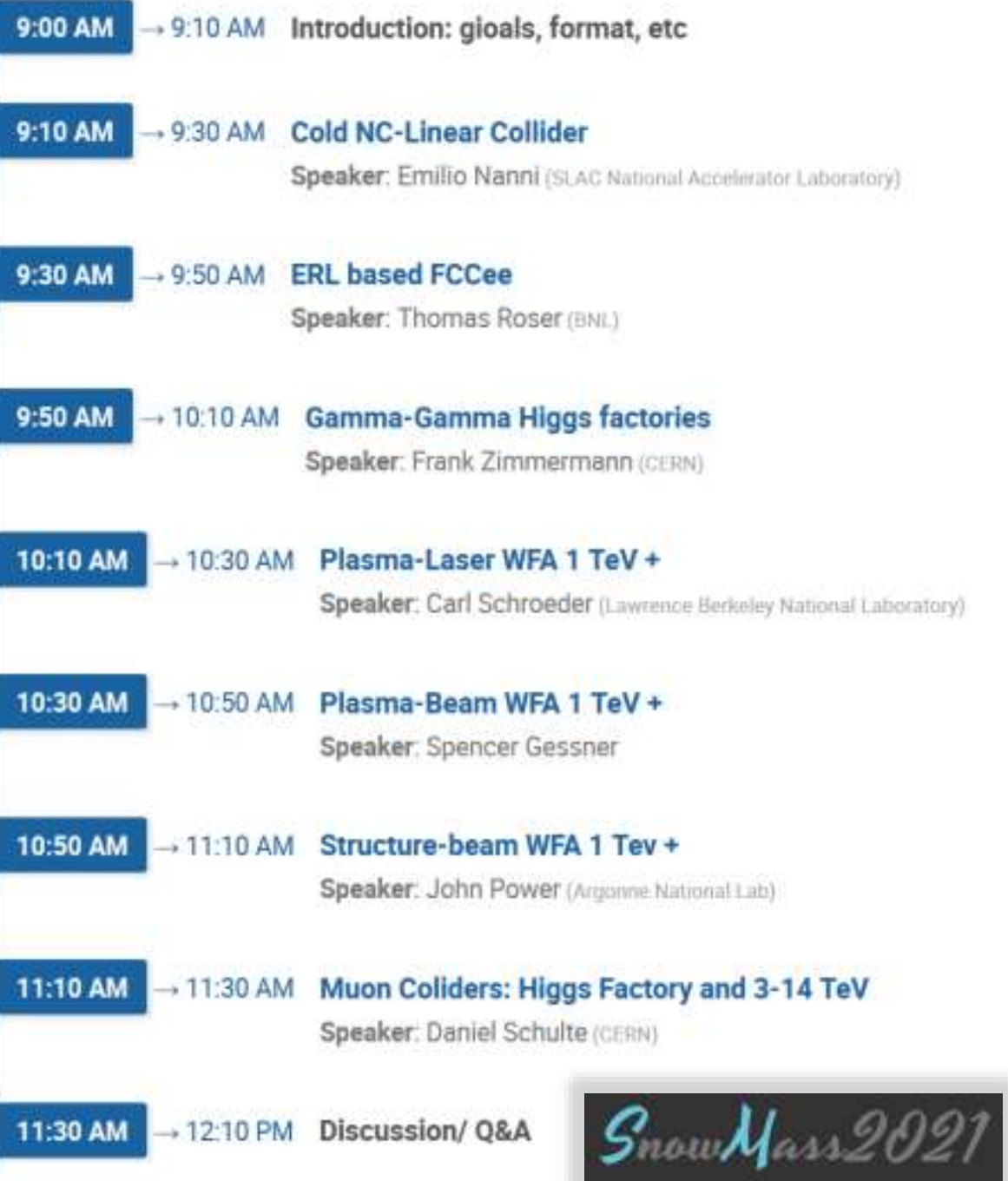




\section{Linear Colliders - Higgs Factories: "Ready"}

International Linear Collider

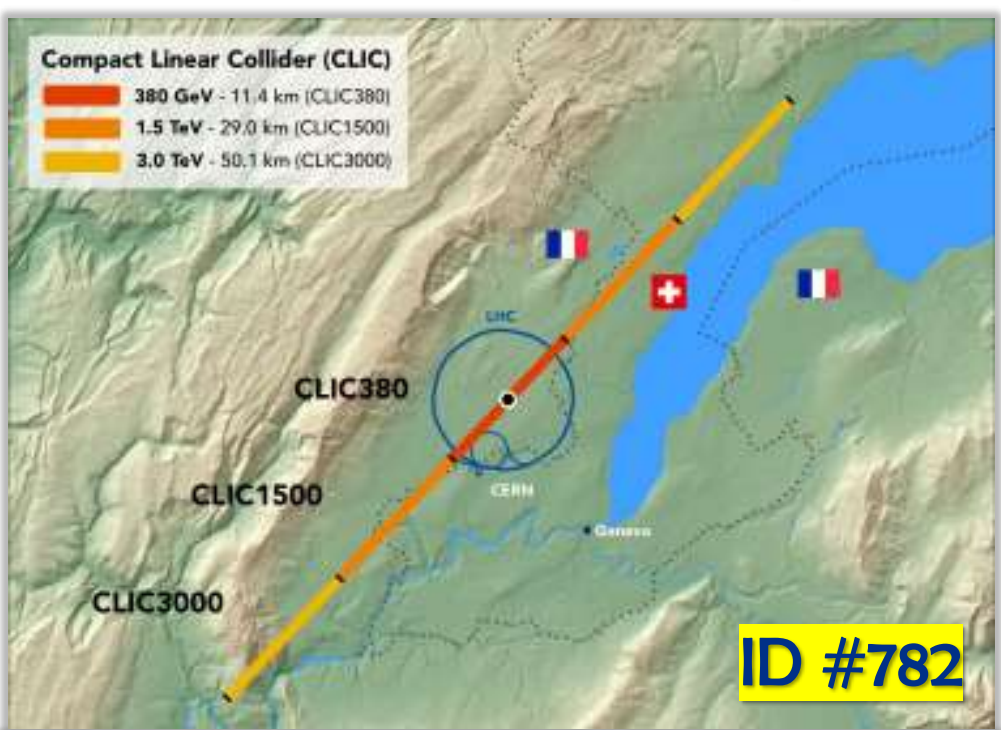

Major R\&D completed

Concerns:

$>$ Positron production (>20x SLC) $\rightarrow$

$>$ Luminosity and commissioning time:

$>$ Ground motion, focusing, etc

$>$ CLIC two-beam scheme is novel

$>$ Option with klystrons as backup

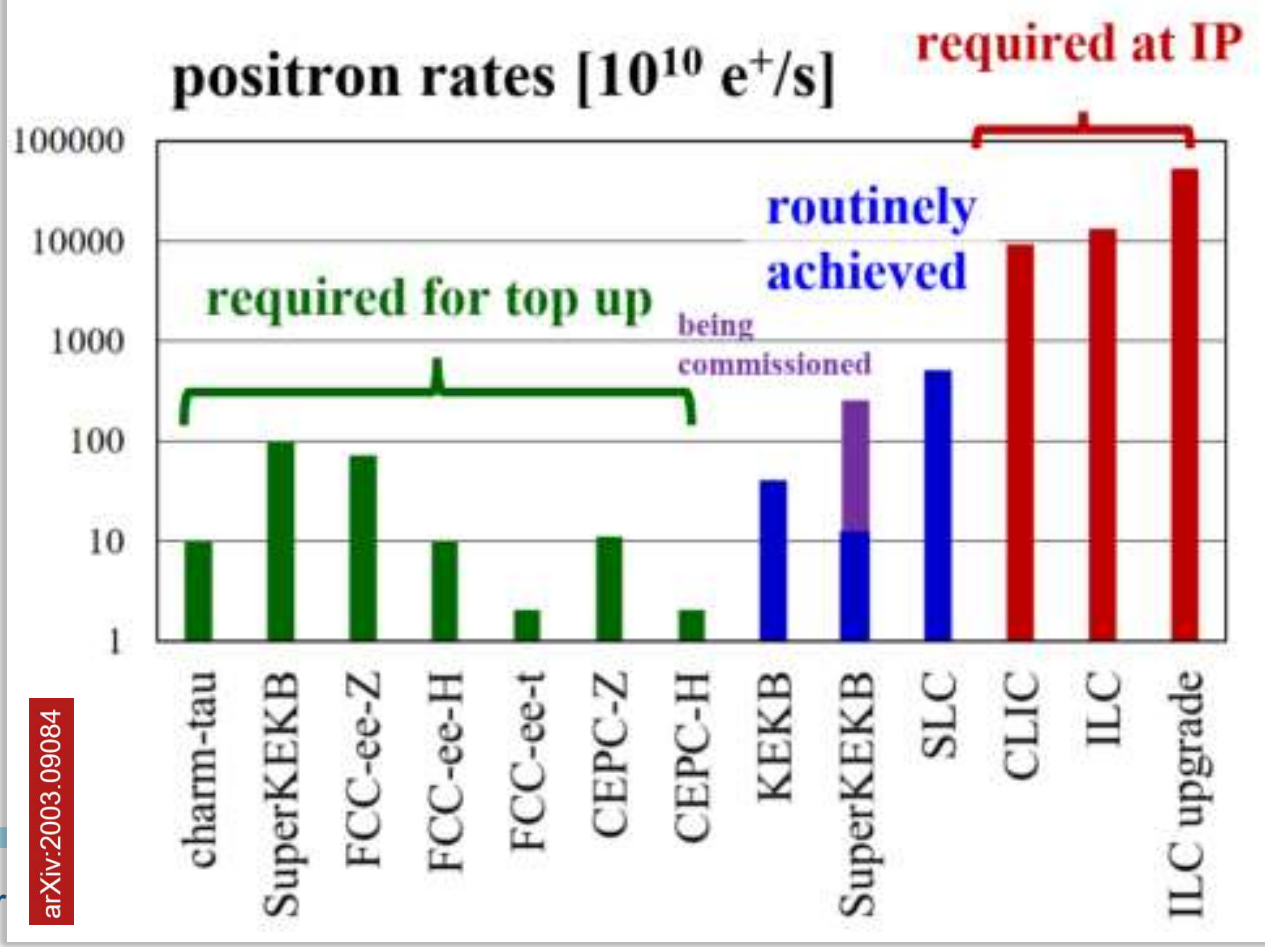




\section{Circular e+e- Higgs Factories: R\&D needed}

\section{FCCee (100km)}

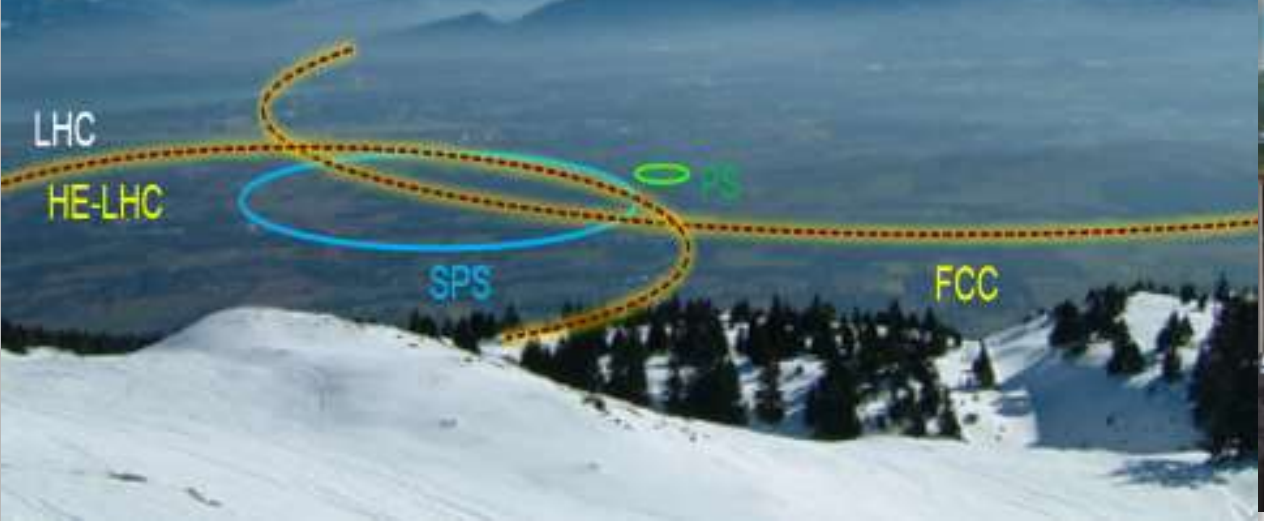

$100 \%$ feasible... matter of cost, time and desired performance/TWh

Challenges:

$>$ Cost reduction

$>$ SRF, magnets, tunnel

$>$ Energy efficiency (now $P \sim 300 \mathrm{MW}$ )

> R\&D collaborations (FCC@CERM)

> CepC TDR by 2023

1) CEPC $650 \mathrm{MHz} 800 \mathrm{~kW}$ high efficiency klystron ( $80 \%$ ) (No 2) High pre
operation) commercial products)

3) CEPC $650 \mathrm{MHz}$ SC accelerator system, including SC caviti and cryomules

4) Collider dual aperture dipole magnets and dual apertur ID \#615, J.Gao et al qudrupoles 


\section{Circular $p p$ Colliders}

HE-LHC CDR (2018) FCC-hh CDR (2018)
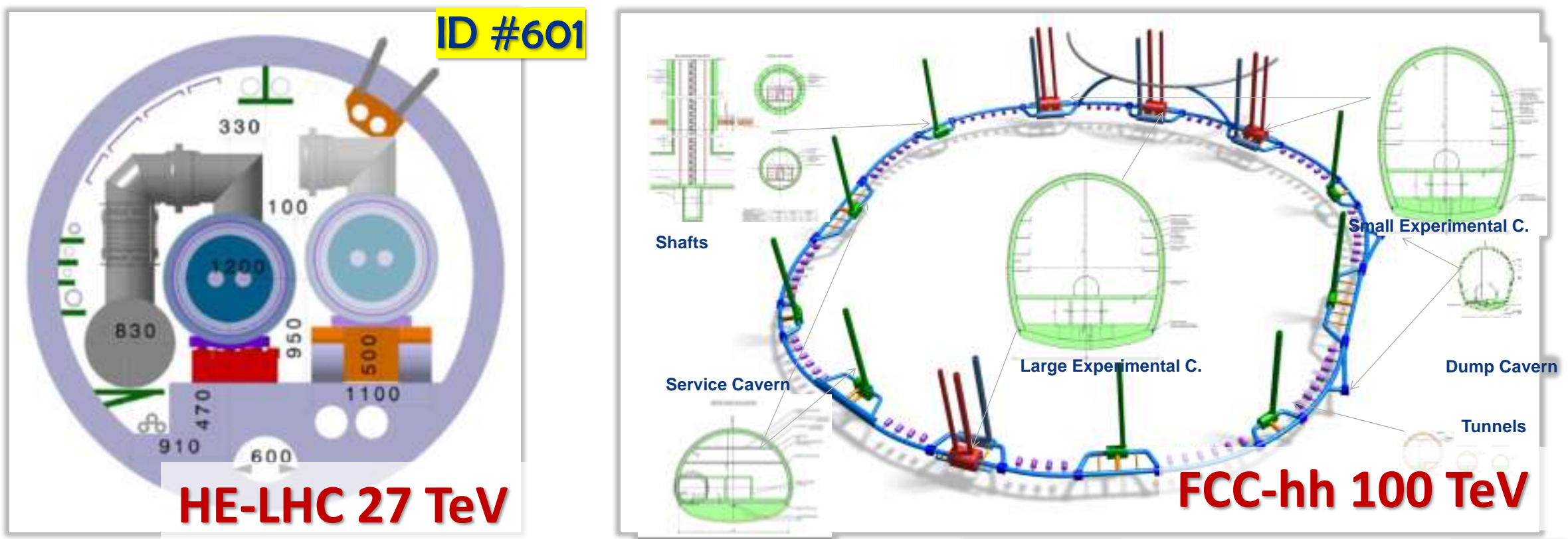

Key facts:

Long tunnels

SC magnets
HE-LHC / FCC-hh* / SppC*

$-27 / 100 / 100 \mathrm{~km}$

$-16 / 16 / 12 \mathrm{~T}$

$\mathrm{O}\left(10^{35}\right) / \mathrm{O}(500)$

$-200 / 500 ? /$ ?

$-7.2 / 17.1 /$ ?

${ }^{*}$ follow up after e+e- Higgs factories

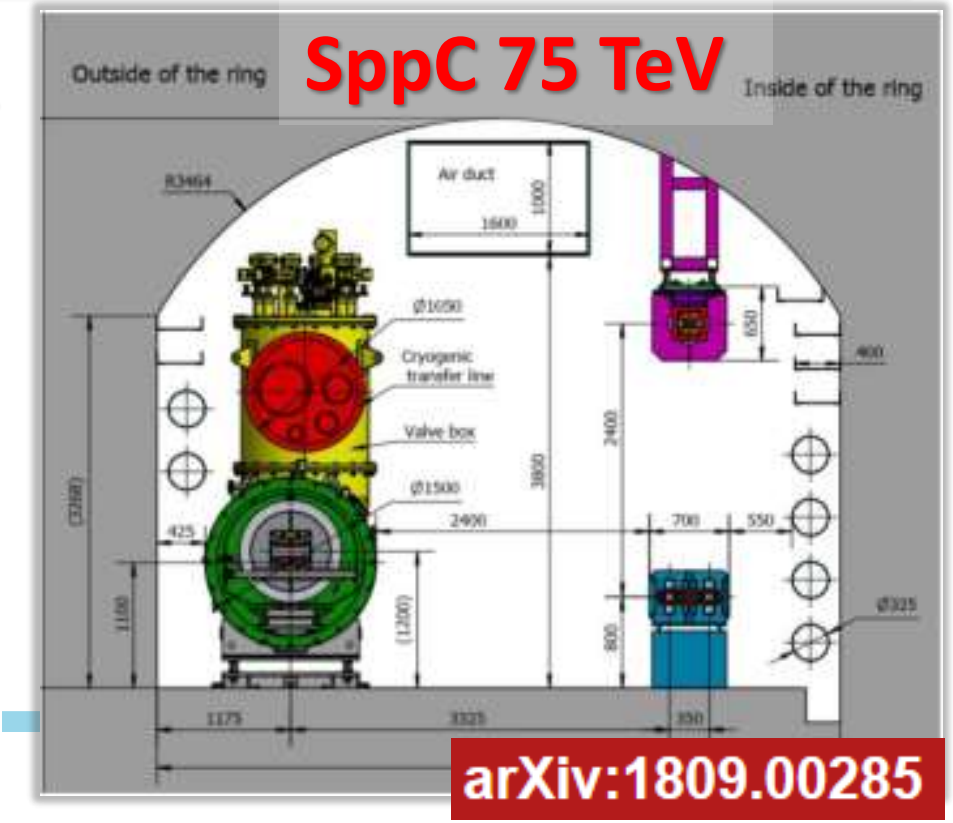




\section{3-14 TeV Muon Colliders: Active R\&D}

ID \#53

ID\#640

$\mu \mu @ 14 \mathrm{TeV} \approx p p @ 100 \mathrm{TeV}$

Ionization cooling of muons demonstrated in MICE @ RAL

ZDRs for $1.5 \mathrm{TeV}, \quad \boldsymbol{\mu}$ Injector

$3 \mathrm{TeV}, 6 \mathrm{TeV}$

and $14 \mathrm{TeV}$

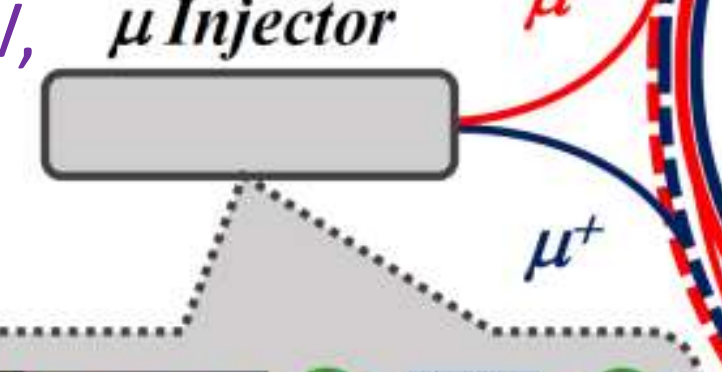

Where is practical limit of the Muon Colliders?

\section{4...30...100 TeV?}

MC is generally within reach of modern accelerator technologies $\mathrm{R} \& \mathrm{D}$ required on: $\mu$ production and cooling, fast acceleration (magnets, RF), MDI, large aperture $12 \mathrm{~T}$ magnets, $v$-radiation Int'I MC-Collaboration - contact D.Schulte(CERN) : $C D R$ in 4 yrs, test facility 6 yrs +4 more years for TDR 


\section{Energy Efficiency of Future Colliders}

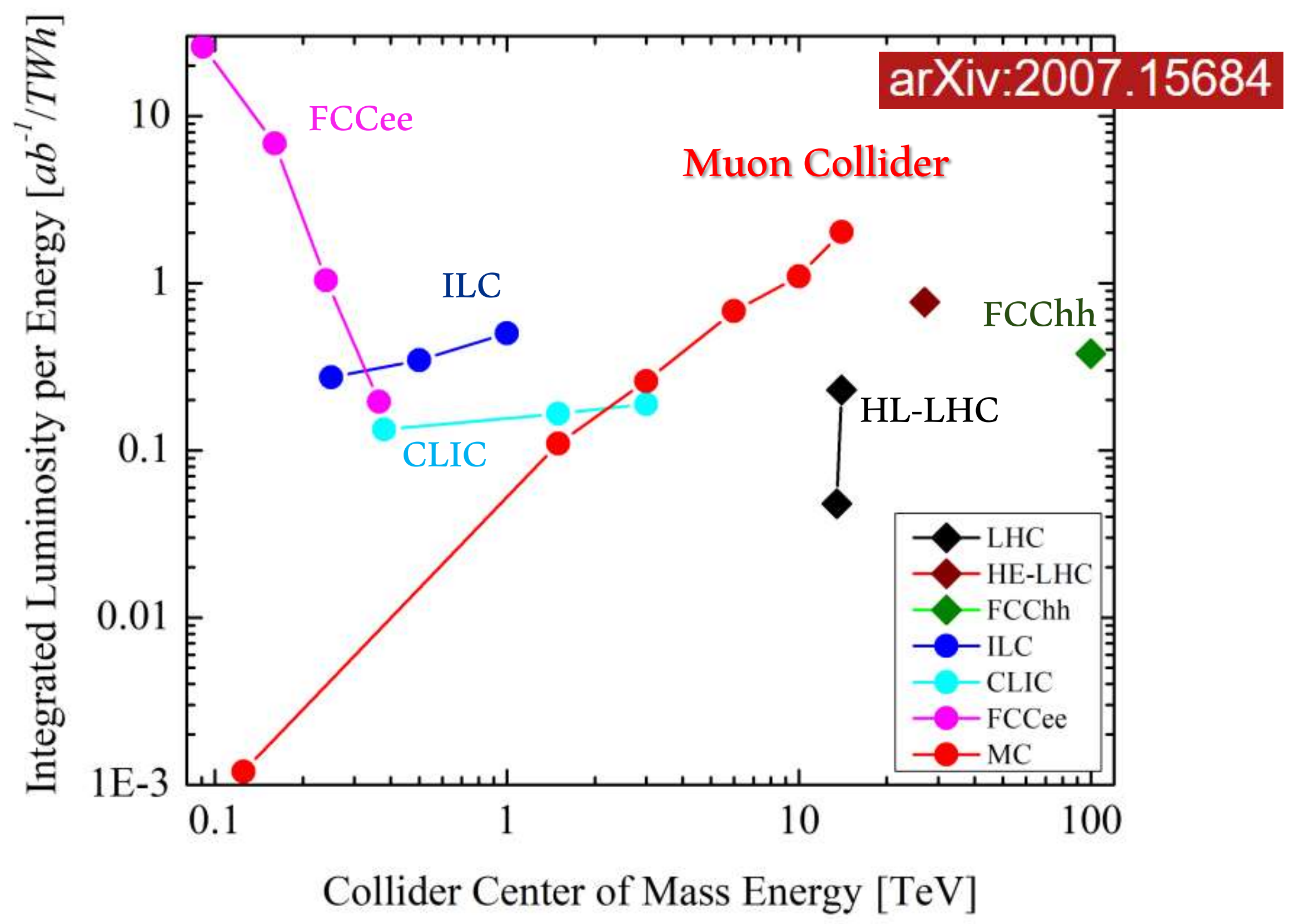




\section{New (Realistic) Ideas}

\section{Energy Recovery Linac (ERL) FCCee:}

$240 \mathrm{GeV}, 100 \mathrm{~km}, 3-10 x$ less RF power $/ \mathrm{ab}^{-1}$ Challenges: emittances, beam-beam, $60 \mathrm{GeV}$ SRF

ERL-based LHeC (or FCCeh):

$60 \mathrm{GeV}$ SRF, $6-9 \mathrm{~km} \quad \sqrt{\mathrm{s}}=1.3 \mathrm{TeV} \sqrt{\mathrm{s}}=3.5 \mathrm{TeV}$

$O\left(1 a b^{-1}\right), 1.6$ BCHF R\&D: ERL PERLE@Orsay

\section{Gamma-Gamma Higgs-Factories:}

Need only $80 \mathrm{GeV}$ e- to reach Higgs, RLA R\&D: design, laser vs FEL, cost, Lumi/TWh

\section{"Gamma-Factory":}

LHC ions + laser $\rightarrow O(\mathrm{GeV}) \gamma^{\prime} \mathrm{s} \rightarrow \mathrm{e}+, \mu$ 's proof-of-principle done; R\&D: high flux

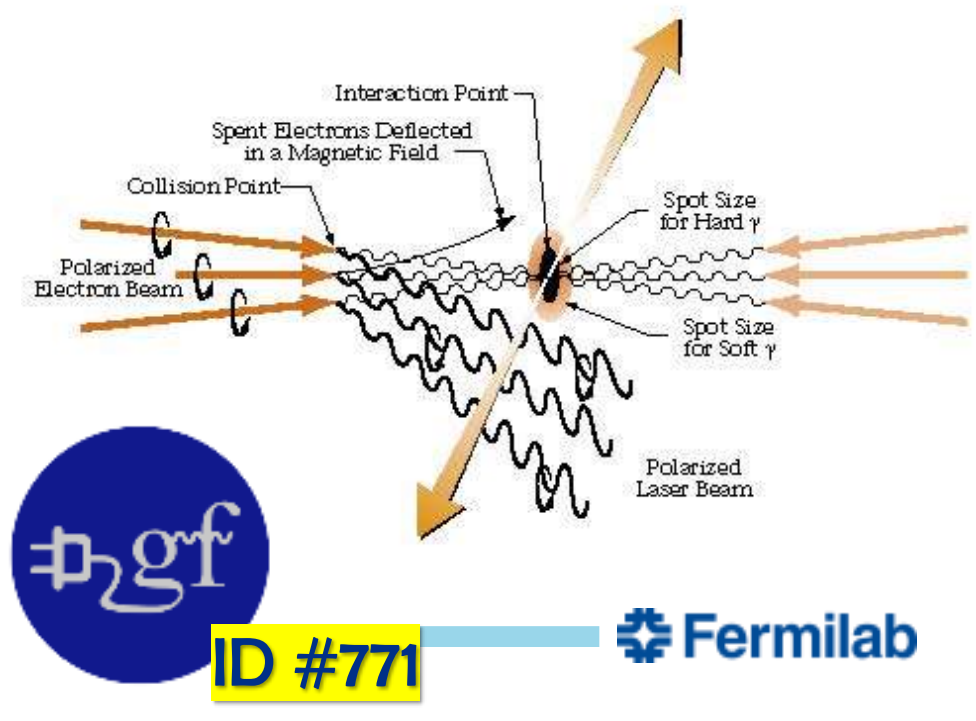

Phys. Lett. B 804

(2020) 13594

ID \#729

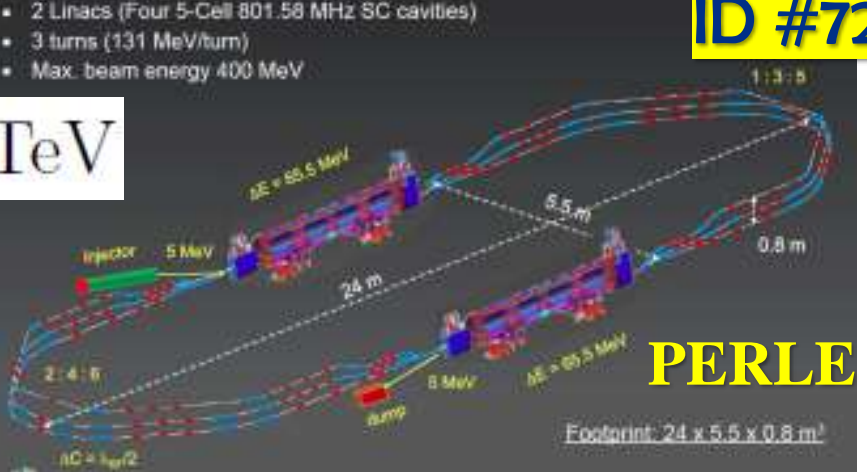




\section{Advanced Accelerator R\&D: Plasma Wakes}

$$
E_{0}=\frac{m_{e} c \omega_{p}}{e} \approx 100\left[\frac{G e V}{m}\right] \cdot \sqrt{n_{0}\left[10^{18} \mathrm{~cm}^{-3}\right]}
$$

Proof-of-principle over past 2 decades:

Three ways to excite plasma (drivers) laser $\quad d E \sim 4.3 \mathrm{GeV}\left(10^{18} \mathrm{~cm}^{-3} 9 \mathrm{~cm}\right)$ $e$ - bunch $d E \sim 9 \mathrm{GeV}\left(\sim 10^{17} \mathrm{~cm}^{-3} 1.3 \mathrm{~m}\right)$ $p+$ bunch $d E \sim 2 \mathrm{GeV}\left(\sim 10^{15} \mathrm{~cm}^{-3} 10 \mathrm{~m}\right)$ In principle, feasible for $e+e$ - collisions, but too early to count - need more R\&D:

- acceleration of positrons, beamstrahlung

- staging efficiency, $E_{\text {max }}$, power efficiency

- emittance control vs vibrations \& scatter Active collaborations focused on applications: ALEGRO, BELLA, FACET-II, EuPRAXIA, ..
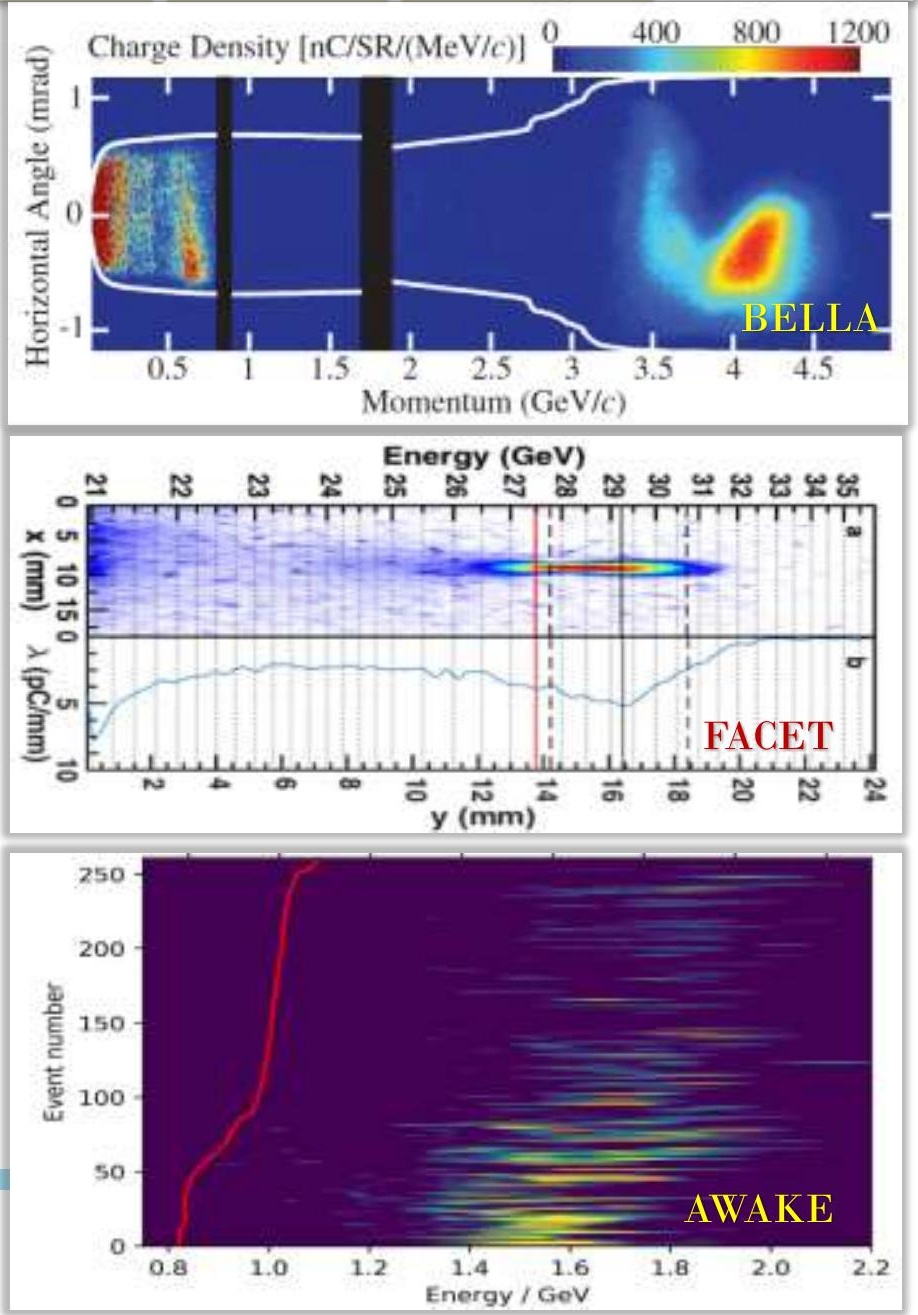
Project

ILC

CLIC

CEPC

FCC-ee

0.24

arXiv:2003.09084

LHeC

HE-LHC

FCC-hh

FCC-eh

Muon Coll

0.25

0.5

1

0.38

1.5

3

0.24

100

3.5

\section{Cost}

4.8-5.3BILCU

8.0 BILCU

$+(\mathrm{n} / \mathrm{a})$

$5.9 \mathrm{BCHF}$

$+5.1 \mathrm{BCHF}$

$+7.3 \mathrm{BCHF}$

5 B USD

$+(\mathrm{n} / \mathrm{a})$

10.5 BCHF

$0.35+1.1 \mathrm{BCHF}$

$1.31 .75^{*} \mathrm{BCHF}$

$27 \quad 7.2 \mathrm{BCHF}$

$17(+7) \mathrm{BCHF}$

$1.75 \mathrm{BCHF}$

$10.7^{*} \mathrm{BCHF}$

\section{Cost - Focused R\&D}

- Many project cost estimates become available at the EPPSU

- Will continue @ Snowmass'21

- "Performance-cost" optimization is critical: e.g. (A.Yamomoto, Granada)

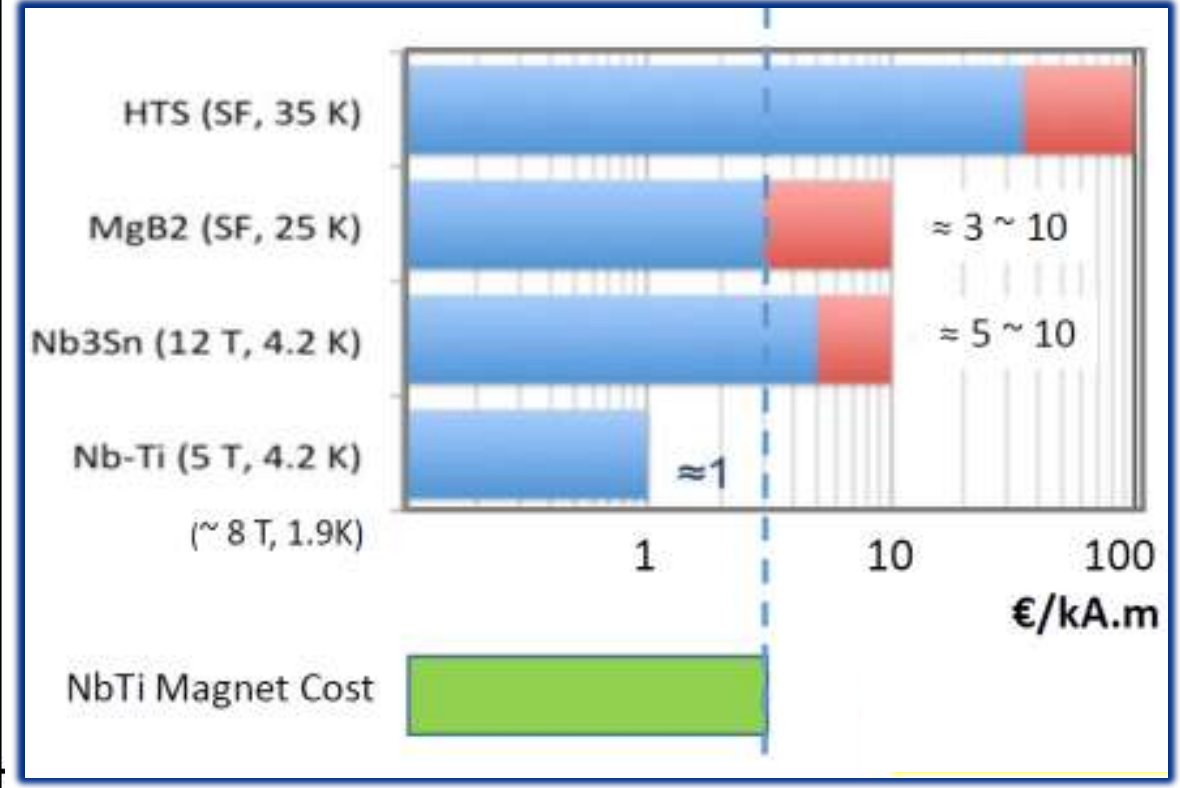

- It takes time to reach the costperformance goals:

$\mathrm{Nb}_{3} \mathrm{Sn}, 12-14 \mathrm{~T}$ : 5-10 yrs short-model R\&D $\mathrm{Nb}_{3} \mathrm{Sn}, 14-16 \mathrm{~T}$ : 10-15 yrs short-model R\&D following by $\sim 10$ yrs for prototype/pre-series (A.Yamomoto, EPPSU, Granada 2019) 


\section{Acknowledgements}

Input/useful discussions with:

\section{arXiv:2003.09084}

Frank Zimmermann (CERN)

\section{"Modern and} Future Colliders"

Steve Gourlay(LBNL)

Tor Raubenheimer (SLAC) Dmitri Denisov (BNL) Meenakshi Narain (UB)

Jorgen D'Hondt (ECFA)
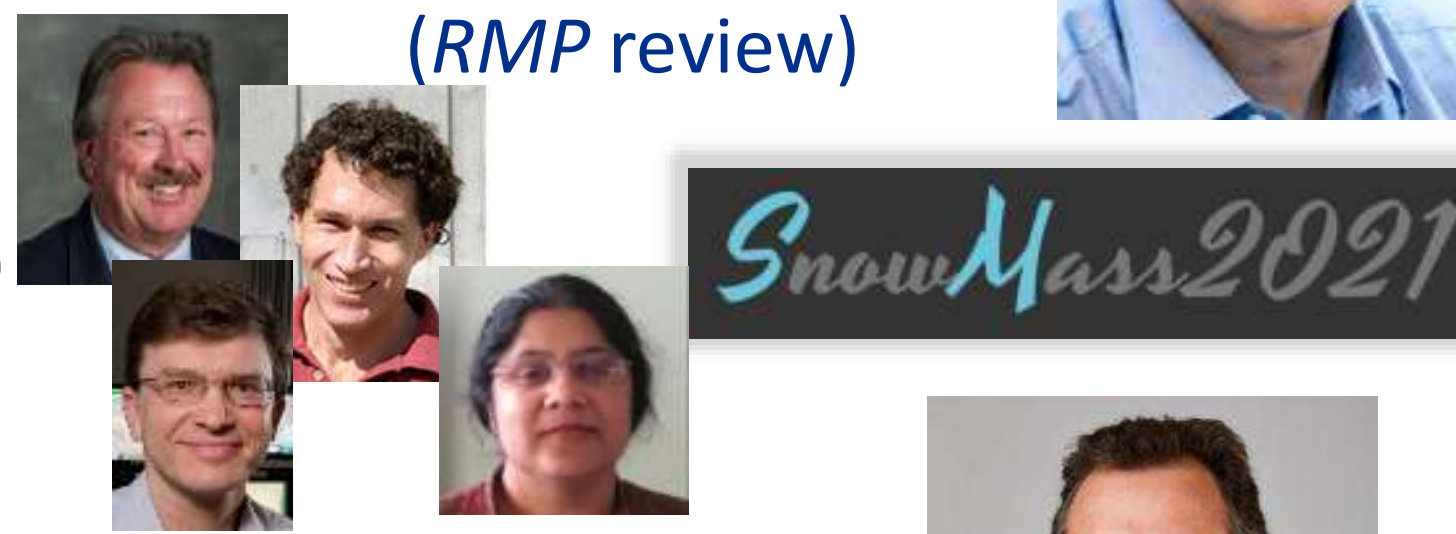

don't miss his plenary talk on Colliders on Wed!

\section{EPPSU-Snowmass'21 input from:}

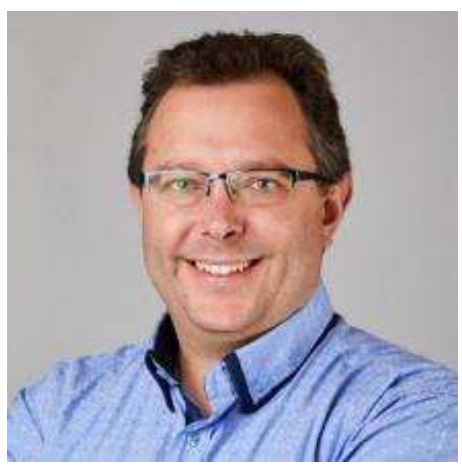

M.Benedikt (CERN), P.Bhat (FNAL), C.Biscari (ALBA), A.Blondel (CERN), J.Brau (UO), O.Bruning(CERN), A.Canepa (FNAL), W.Chou (IHEP, China), J.P.Delahaye (CERN), D.Denisov (BNL), V.Dolgashev (SLAC), E.Gschwendtner (CERN), A.Grasselino (FNAL), M.Klein (CERN), W.Krasny (CNRS), M.Lamont (CERN), W.Leemans (DESY), E.Levichev (BINP), K.Long (ICL), D.Luccessi (INFN), B.List (DESY), H.Montgomery (JLab), P.Muggli (MPG), D.Neuffer (FNAL), H.Padamsee (Cornell), M.Palmer (BNL), N.Pastrone (INFN), Q.Qin (IHEP), L.Rivkin (EPFL/PSI), A.Romanenko (FNAL), T.Roser (BNL), M.Ross (SLAC), D.Schulte (CERN), A.Seryi (Jlab), T.Sen (FNAL), A.Valishev (FNAL), F.Willeke (BNL), A.Yamomoto (KEK), V.Yakovlev, A.Zlobin (FNAL 춫 Fermilab 


$$
\begin{aligned}
& \text { Thank You for } \\
& \text { rour Attention! }
\end{aligned}
$$




\section{BACK UP SLIDES}




\section{Summary:}

- Remarkable progress of the projects/proposals/technologies:

- esp. ILC, CLIC, FCC-ee, - $h$, CepC, $\mu$-Colliders, plasma, ...

- allow in-depth evaluation of readiness, power and costs

- Higgs Factories Implementation :

- several feasible options on the table

- the choice might define high-energy future collider choice

- Highest Energy Future Colliders:

- demand very high AC power \& cost; some options to save

- each machine has a set of key R\&D items for next 7-10 yrs

- core acceleration technology R\&D - SC magnets, SRF and plasma - are of general importance and help all - pp/ee/ $\mu \mu$

- We also expect to gain valuable experience from the machines to be built and operated over the next decade

- (see next slide) 


\section{Country}

\section{Experience}

SuperKEKB Japan 7+4 Gev e+e-,

$8 \mathrm{e} 35$

nano-beams

scheme

$H L-L H C$

CERN

x5 LHC luminosity

$\mathrm{Nb}_{3} \mathrm{Sn}$ magnets,

crab cavities

NICA

Russia

ii/pp 11-27 GeV

electron and stochastic cooling

PIP-II

USA

SRF linac to

double \# v's

CW SRF, >1 MW

targetry

ESS

Sweden

$5 \mathrm{MW}$ pulsed SRF

SRF, cryo, targetry

LCLS-II-HE USA

$8 \mathrm{GeV}$ CW SRF

efficient SRF, cryo

SuperC-Tau Russia

2-6 GeV e+e-

crab waist scheme

EIC

USA

20-140 GeV ep/ei polarization, cool'g 


\section{EPPSU and Snowmass'21}

CERN Council Open Symposium on the Update of

\section{European Strategy for Particle Physics}

13-16 May 2019 - Granada, Spain

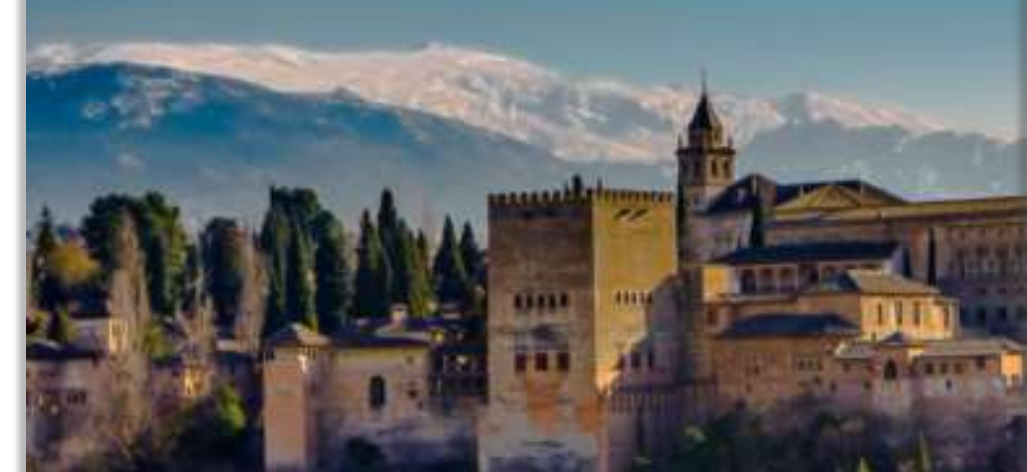

https://cafpe.ugr.es/eppsu2019/

https://snowmass21.org/

European Strategy

CERN-ESU:-204
30 Sopienter 2019

Physics Briefing Book

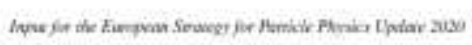

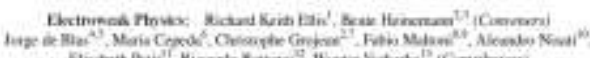

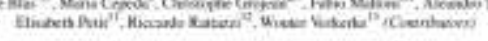

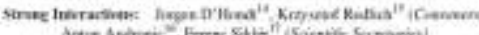

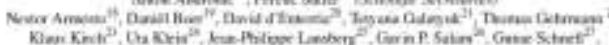

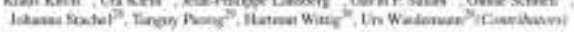

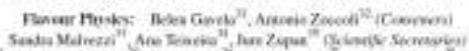

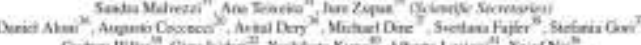

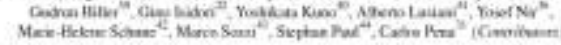

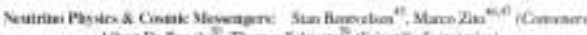

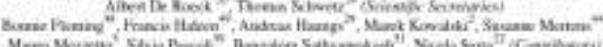

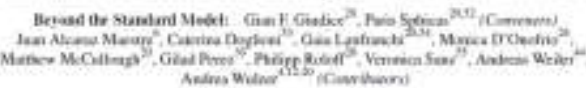

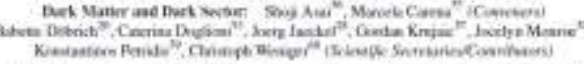

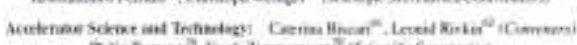

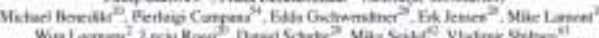

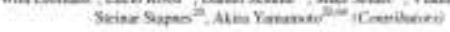

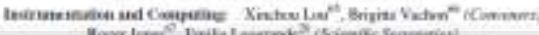

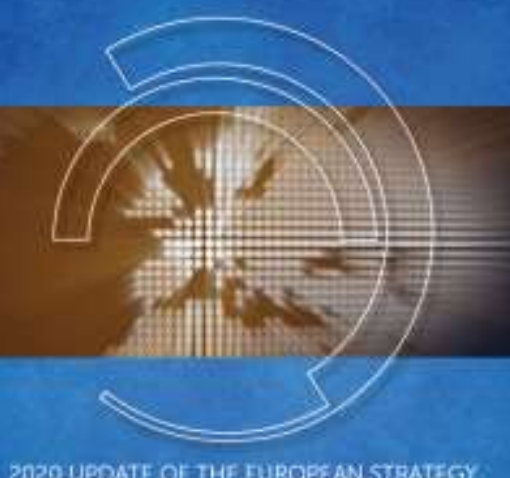

2020 UPQATE OF THE EUROPEAN STRATEG
FOR PARTCLE PHYSICS

by the European Strategy Group

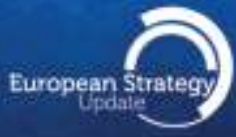

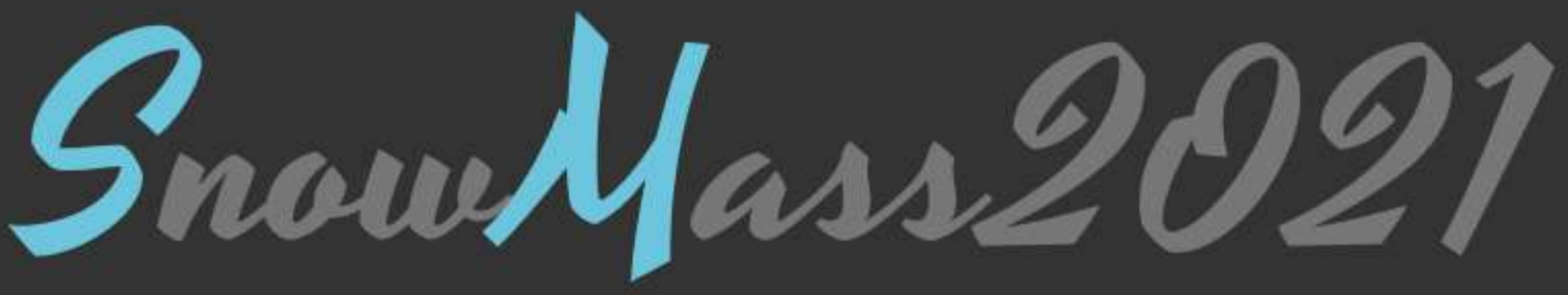

ilab 
The cost for the machine alone is about 4.6 billion CHF (about 3 billion Euro). The total project cost breaks down roughly as follows:

\begin{tabular}{|l|c|c|c|}
\hline $\begin{array}{l}\text { Construction costs } \\
\text { (BCHF) }\end{array}$ & Personnel & Materials & Total \\
\hline LHC Machine and areas & 0.92 & 3.68 & $\left.4.60^{\circ}\right)$ \\
\hline $\begin{array}{l}\text { CERN share to } \\
\text { Detectors }\end{array}$ & 0.78 & 0.31 & 1.09 \\
\hline $\begin{array}{l}\text { LHC injector } \\
\text { upgrade }\end{array}$ & 0.09 & 0.07 & 0.16 \\
\hline $\begin{array}{l}\text { LHC computing (CERN } \\
\text { share) }\end{array}$ & 0.09 & 0.09 & 0.18 \\
\hline Total & 1.88 & 4.15 & 6.03 \\
\hline
\end{tabular}

") (inctuding 0.43 BCHF of in-kind contributions)

\section{CERN-Brochure-2008-001-Eng}

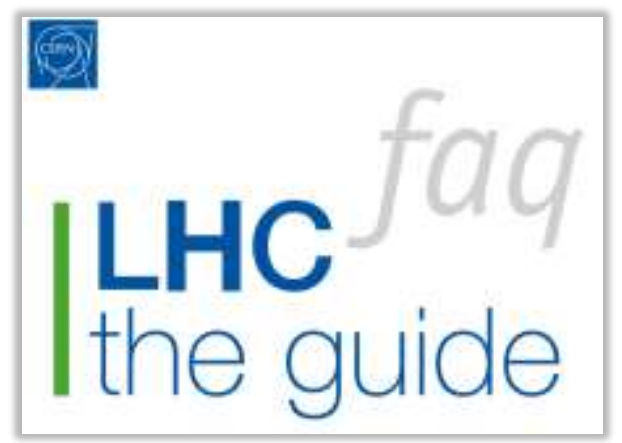

\section{Cost of the LHC}

\section{CERN-Brochure-2017-002-Eng}

huch does it cost?

he total cost for the LHC, detectors and computing is as follows:

\begin{tabular}{|l|c|}
\hline & $\begin{array}{l}\text { Material costs } \\
\text { (MCHF) }\end{array}$ \\
\hline $\begin{array}{l}\text { LHC machine and } \\
\text { areas }\end{array}$ & 3756 \\
\hline $\begin{array}{l}\text { (ERN share to detectors } \\
\text { and detector areas }{ }^{*} \text { ) }\end{array}$ & 493 \\
\hline $\begin{array}{l}\text { LHC computing (CERN } \\
\text { share) }\end{array}$ & 83 \\
\hline Total & 4332 \\
\hline
\end{tabular}

*) This includes: Machine R \& D and injectors, tests and pre-operation.

**) Contains infrastructure costs (such as caverns and facilities). The total cost of all LHC detectors is about 1500 MCHF. 